\title{
Anatomic and terminological description and processing of breast pathologic specimens from oncoplastic large volume displacement surgeries
}

\author{
Yuanxin Liang ${ }^{1} \cdot$ Stephen $\mathrm{P}$ Naber ${ }^{1} \cdot$ Abhishek Chatterjee $^{2}$
}

Received: 10 October 2017 / Revised: 14 December 2017 / Accepted: 17 December 2017 / Published online: 15 February 2018

(c) United States \& Canadian Academy of Pathology 2018

\begin{abstract}
Oncoplastic surgery provides breast cancer patients with greater aesthetic satisfaction without compromising disease-free survival or overall survival rate. Large volume displacement oncoplastic surgical techniques have become increasingly popular as a strategy for improving aesthetic outcomes and extending the option of breast conservation therapy. They often involve breast reduction or mastopexy reconstructive techniques to facilitate resection of large breast volumes on the side of the breast cancer and accompanied with symmetry contralateral breast reductions or mastopexies. However, dissection of large volume displacement oncoplastic surgical specimens presents unique challenges. Compared with traditional mastectomy specimens, they are relatively complicated, which requires the pathologist to understand the surgical procedure and the anatomy of the specimens. Given this, we introduce the standard anatomical and terminological description for the breast pathologic specimens of five large volume displacement oncoplastic surgical techniques commonly performed in our institution for breast cancer management. The individual surgical specimen is composed of one or several components, which include lateral wall, superior keyhole, medial wall, lateral wing, inferior pole, and medial wing. We also present specimen documentation and sectioning procedures used in our institution. The advantages for the patient provided by large volume reduction oncoplastic surgery must be supported by proper evaluation of the surgical pathology specimen. Therefore, we recommend that each section taken from the oncoplastic specimen be labeled as to its specific location in the specimen components. Standardized nomenclature and technique will assist pathologists in accurately evaluating the surgical margins.
\end{abstract}

\section{Introduction}

Breast cancer has been the most frequently diagnosed cancer among women for decades; $12.3 \%$ of women will be diagnosed with breast cancer at some point during their lifetime [1]. Surgical management of breast cancer has trended away from radical mastectomy toward breastconserving techniques. Oncoplastic surgery has steadily gained popularity, initially in Europe where it was first described, and more recently in the United States where it continues to increase in popularity with increasing

Stephen P Naber

snaber@tuftsmedicalcenter.org

1 Department of Pathology and Laboratory Medicine, Tufts Medical Center, Boston, MA, USA

2 Department of Surgery, Tufts Medical Center, Boston, MA, USA representation in the breast cancer surgical literature [2]. Oncoplastic surgery provides patients with greater aesthetic satisfaction without compromising disease-free survival or overall survival rate [3, 4]. Large volume displacement oncoplastic surgery is the excision of a large lumpectomy and subsequent manipulation of local breast tissue, often using a breast lift or breast reduction plastic surgery design to reconstruct the void of tissue created by the large lumpectomy. Often a contralateral symmetry breast lift is needed so that the aesthetic and functional outcome of both breasts is optimized [5]. Multiple oncoplastic techniques have been developed as oncoplastic breast-conserving surgery was introduced by Audretsch in 1998 [6-8]. Large volume displacement oncoplastic surgical techniques are associated with high rates of successful breast conservation and low rates of required re-excisions compared with standard lumpectomy [4, 9]. In the suitable breast cancer patient, oncoplastic surgery has also shown to be costeffective [10]. It has become increasingly popular as a 
strategy for improving aesthetic outcomes and extending the option of breast conservation therapy [11]. These multifaceted benefits with oncoplastic surgery have led to an increase in its popularity as a breast conservation option. Subsequently, rates of oncoplastic surgery in major institutions have increased and have been reported to be as high as $34 \%$ of all breast conservation operations performed in institution offering oncoplastic surgery as a breastconserving option. [12]

Postoperative pathologic diagnosis of a breast surgical specimen is usually considered the gold standard to provide the basis for clinical treatment and management decisions. Timely and proper gross dissection of oncologic breast specimens is important for surgical margin evaluation, tumor grading, staging, biomarker measurement, and chemotherapeutic regimen selection for breast cancer. However, dissection of large volume displacement oncoplastic surgical specimens presents unique challenges. Compared with traditional mastectomy specimens, they are relatively complicated; which requires the pathologist to understand the surgical procedure and the anatomy of the specimens. Large volume displacement oncoplastic surgery inherently excises larger volumes of tissue compared with standard lumpectomy [9]. In addition, the skin incision patterns often do not overlie the area of cancer leading to further potential confusion and miscommunication between what the surgeon sees and the pathologist perceives. Unfortunately, there is no literature in pathology systematically describing breast oncoplastic specimen processing. The growth of large volume displacement oncoplastic breast surgery introduces a new class of specimens for pathologists to evaluate. The traditional approach of serially sectioning a breast excision or mastectomy specimen has been the standard approach for evaluating the tumor and the surgical margins. This works very well for specimens, which can be easily oriented and the margins clearly defined as to their anatomic location. However, many of the specimens generated from breast oncoplastic surgery have unique shapes and some may consist of multiple parts that may or may not be contiguous with one another. Consequently, pathologists must now become familiar with this class of breast specimens and develop a consistent strategy for their pathologic examination in addition to communicating with the oncoplastic surgeon with regards to the orientation of the specimen(s). The first step is for pathologists to learn the proper nomenclature for the various oncoplastic specimens and their components and then to adopt uniform procedures for evaluating the tumor and surgical margins and documenting the location of histologic sections. Therefore, our goal is to: [1] describe the standard anatomy and terminology for the breast pathologic specimens of large volume oncoplastic surgeries performed at our institution; [2] describe the procedure for processing large volume displacement oncoplastic specimens; [3] discuss outcomes from our large volume displacement oncoplastic single institution registry highlighting the importance of specimen processing.

Herein, we describe the most common large volume displacement oncoplastic surgeries performed at our institution. These are well documented in the breast oncology and plastic surgery literature when discussing oncoplastic surgery. The surgical terminology used in the descriptions within this paper are found in the general breast reconstructive literature [4, 6, 8, 13-17]. We also propose a standard approach to process these oncoplastic breast specimens.

\section{Understanding the individual surgical procedure and pathologic specimens}

There are five large volume displacement oncoplastic surgical techniques commonly performed in our institution for breast cancer management. Each technique inherently has [1] a pedicle design, which designates how blood is supplied to the nipple areola complex, and [2] a skin pattern design that describes the type of incision pattern that would facilitate a breast lift or reduction.

\section{Superior pedicle circumvertical skin incision pattern oncoplastic surgery}

The superior pedicle designates the (Fig. 1a) blood supply to the nipple areola complex to come from mostly the thoracoacromial and lateral thoracic branches [18]. The circumvertical skin incision pattern appears as a "snowman" design with the superior circle just above the nipple areola complex and the inferior circle encompassing the region of the inferior pole that is to be excised. The superior circle is de-epithelialized with the nipple areola complex eventually pulled superiorly and the lateral and medial walls brought inferiorly and centrally to fill in the region of the inferior circle hence giving the patient a breast lift. The breast specimen is derived from the inferior portion of the breast and is termed an inferior pole specimen. The superior margin of this specimen is therefore adjacent to the inferior edge of the pedicle.

\section{Superomedial pedicle circumvertical skin incision pattern oncoplastic surgery}

The superomedial pedicle designates the blood supply to (Fig. 1b) the nipple areola complex to come from internal mammary perforators [18]. The circumvertical skin incision pattern appears as a snowman design with the superior circle just above the nipple areola complex and the inferior circle encompassing the region of the inferior pole that is to 

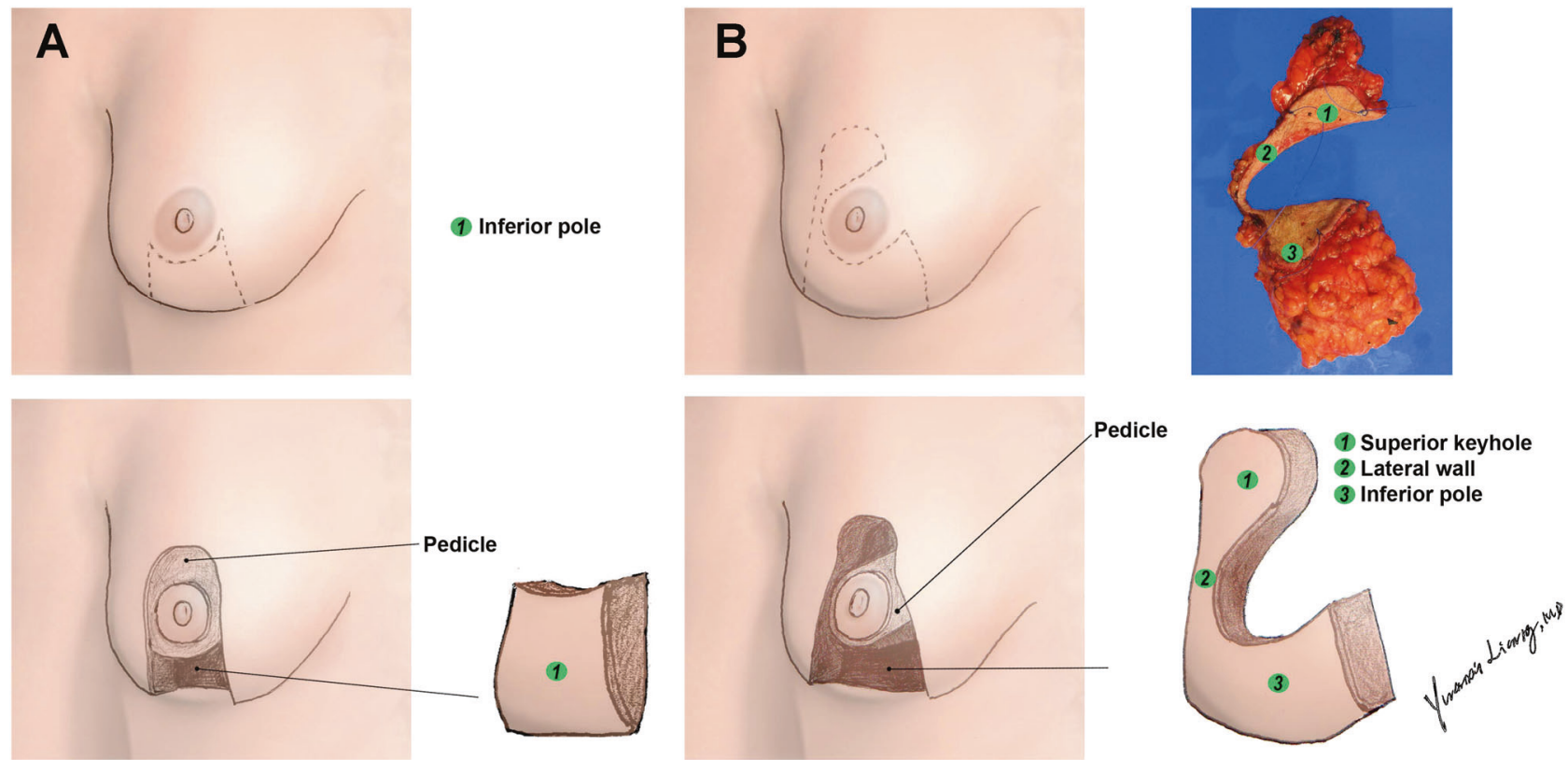

Fig. 1 Breast specimens of circumvertical skin incision pattern. The skin excision patterns are represented on the breast drawing by dotted lines. a superior pedicle circumvertical skin incision pattern. The shaded de-epithelialized area surrounding the nipple areola is not part of the surgical specimen. The single specimen is from the inferior pole with its superior edge representing the inferior margin of the pedicle. b superomedial pedicle circumvertical skin incision pattern. The surgical specimen has its three contiguous components (superior keyhole, lateral wall, inferior pole) in a single specimen. The pedicle margins are represented by the inferior edge of the superior keyhole, the medial edge of the lateral wall, and the superior edge of the inferior pole. No pathologic specimen is sent from pedicle be excised. The superomedial pedicle is de-epithelialized around the nipple areola complex. Part of the lateral wall, the inferior pole, and part of the superior keyhole are regions of tissue that are excised. Finally, the lateral and medial walls are brought inferiorly and centrally and the superomedial pedicle with the nipple areola complex is moved superiorly into the superior keyhole defect. The resulting specimen, taken from middle to inferior portion of the breast, has three components including superior keyhole, lateral wall, and inferior pole. The superior margin is the edge of the keyhole and the middle part of medial margin is adjacent to the pedicle.

\section{Superomedial pedicle inverted-T (Wise) skin incision pattern oncoplastic surgery}

Wise-pattern breast reduction remains the most popular (Fig. 2a) method of performing moderate- to large-sized breast reductions in the United States [19]. The superomedial pedicle designates the blood supply to the nipple areola complex to come from internal mammary perforators [18]. The inverted-T skin incision pattern appears as a semicircle above the nipple areola complex with wings spreading inferiorly and laterally. The superomedial pedicle is deepithelialized around the nipple areola complex. Part of the lateral wall, the inferior pole, and part of the superior keyhole are regions of tissue that are excised. Finally, the lateral and medial walls brought inferiorly and centrally and the superomedial pedicle with the nipple areola complex is brought superiorly into the superior keyhole defect. The breast specimen is from the lateral-inferior-medial portion of the breast in addition to the superior keyhole; it is composed of five components including superior keyhole, lateral wall, lateral wing, inferior pole, and medial wing. The medial part of superior margin is adjacent to the pedicle and the lateral part of superior margin is the edge of the keyhole.

\section{Extended superomedial pedicle inverted-T (Wise) skin pattern oncoplastic surgery}

The vertical scar superior-medial pedicle reduction (Fig. 2b) pattern is a versatile oncoplastic technique that allows breast tissue rearrangement for various tumor locations [20]. From a surgical technique standpoint, this is identical to the superomedial pedicle inverted-T skin incision pattern oncoplastic surgery except that the inferior pole is also included and de-epithelialized with the superomedial pedicle. Typically, this additional tissue is rotated into the upper outer quadrant where a large lumpectomy is performed first and the extended superomedial pedicle fills the void created. The breast specimen consists of a medial part and a lateral part. The medial part is composed of medial wing; and the lateral part is composed of three 

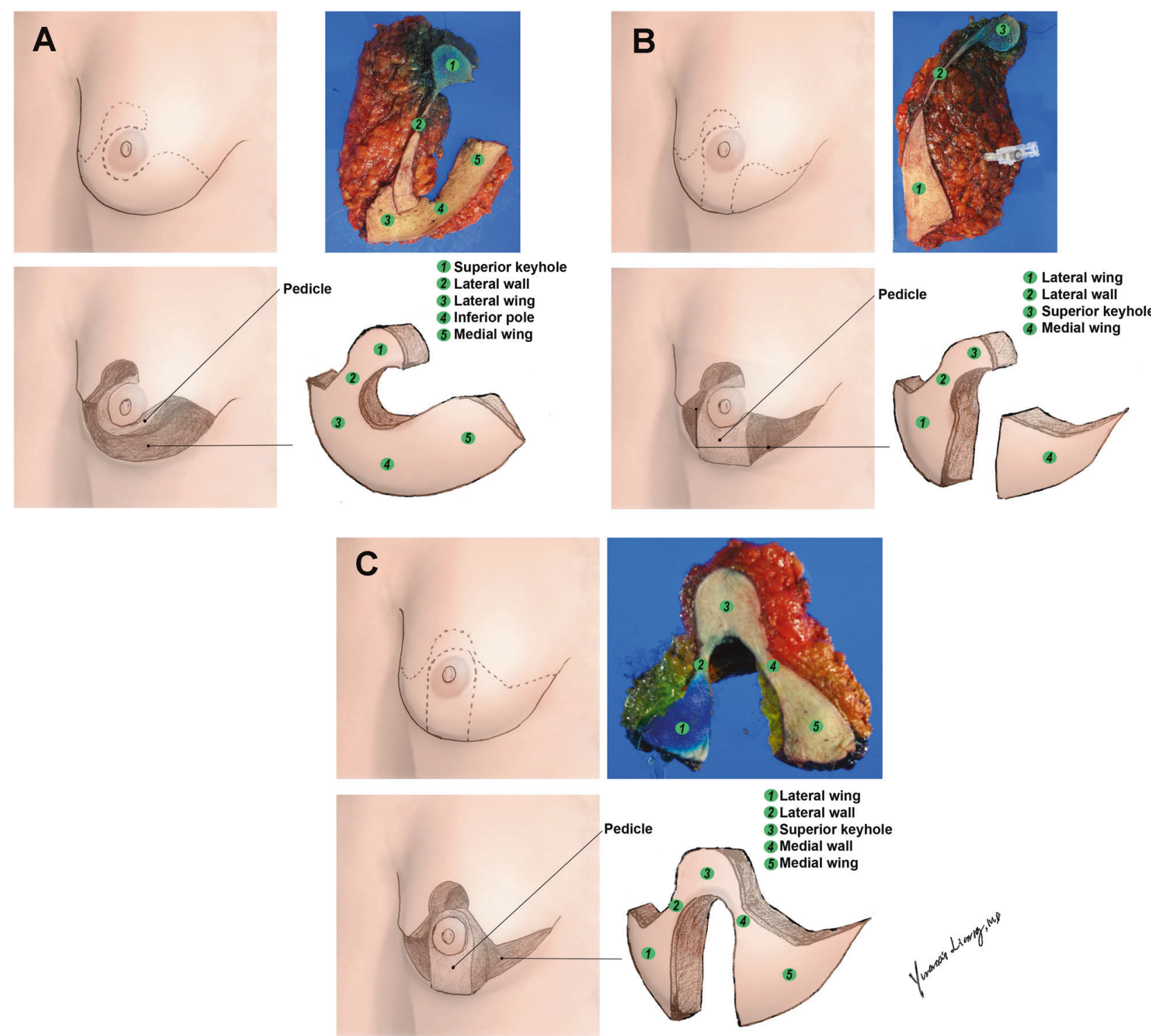

Fig. 2 Breast specimens of inverted-T (Wise) skin pattern. a superomedial pedicle inverted-T (Wise) skin incision pattern. All five components are contiguous in a single specimen. $\mathbf{b}$ extended superomedial pedicle inverted-T (Wise) skin pattern. The medial wing is received as a separate specimen and its lateral edge is related to the

components including part of the superior keyhole, lateral wall, and lateral wing. The superior margin of lateral part is the edge of the keyhole. The medial margin of lateral part and the lateral margin of medial part are adjacent to the pedicle.

\section{Inferior pedicle inverted-T (Wise) skin pattern oncoplastic surgery}

The inferior pedicle designates the blood supply to the nipple areola complex (Fig. 2c) to come from intercostal artery perforators [18]. The inverted-T skin incision pattern appears as a semi-circle above the nipple areola complex medial aspect of the inferior pole. $\mathbf{c}$ inferior pedicle inverted-T (Wise) skin pattern. All components of the specimen are contiguous. The blue area on the skin surface is the sentinel lymph node dye injection site. The tumor is located in this region and the medial aspect of the lateral wing is the margin against the inferior pedicle

with wings spreading inferiorly and laterally. The inferior pedicle is de-epithelialized around and inferior to the nipple areola complex. The lateral wing, superior keyhole, and medial wing are regions of tissue that are excised. Finally, the lateral and medial walls are brought inferiorly and centrally and the inferior pedicle with the nipple areola complex is moved superiorly into the superior keyhole defect. The breast specimen is from the lateral to medial portion of the breast; it is composed of five components including lateral wing, lateral wall, superior keyhole, medial wall, and medial wing. The middle part of superior margin is the edge of the keyhole and the middle part of inferior margin is adjacent to the pedicle. 


\section{Summary of the specimens}

The location of the tumor is the most important factor in choosing a surgical technique and a pedicle location. An inferiorly based pedicle is used if the tumor is located in the upper breast, and the superiorly based pedicle is used if it is located in the lower breast [13]. However, no matter what surgical techniques are chosen, the surgical specimen is always composed of one or several components of lateral wall, superior keyhole, medial wall, lateral wing, inferior pole, and medial wing. The surgeon should ideally do his best to send specimens in continuity including the skin to make it easier upon the pathologist to understand tissue orientation relative to the area of cancer. Nevertheless, specimens may come in separate pieces and using the standard terminology to describe the oncoplastic specimen creates a common language decreasing misunderstanding between the breast cancer treatment team members. Breast tumor can be located in any of those components. The individual components and their relationship to the breast are illustrated in Fig. 3. We recommend that each section taken from the oncoplastic specimen should be labeled by its location within those components.

\section{Specimen receipt and documentation}

The pathologic examination of large volume reduction oncoplastic surgical specimens can be more complicated

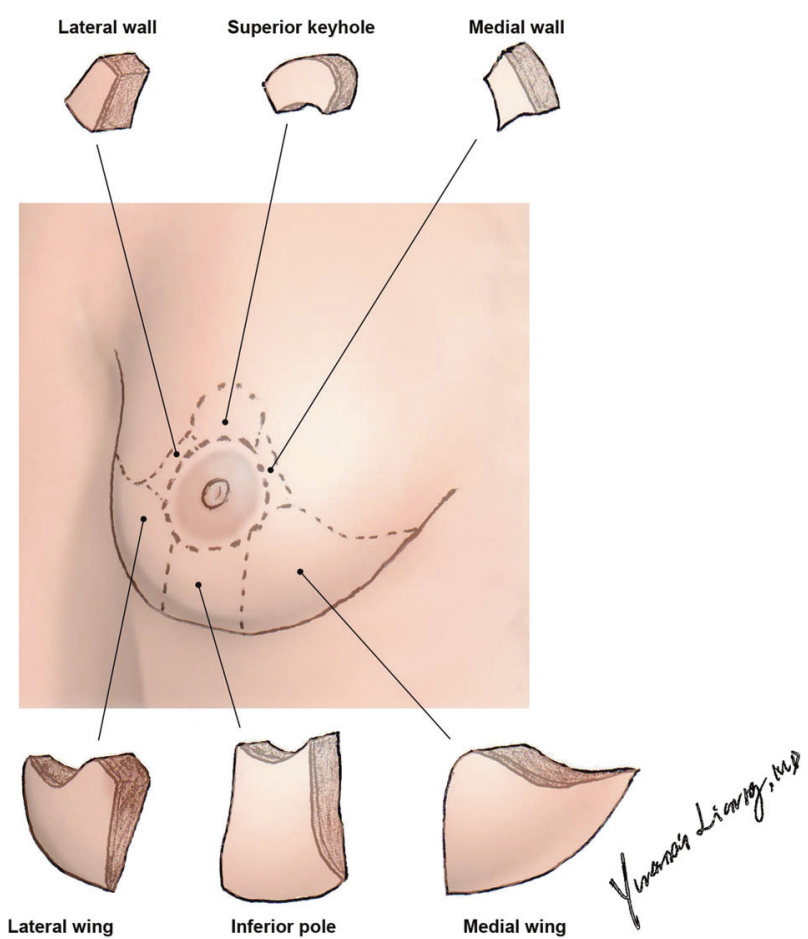

Fig. 3 The individual components of oncoplastic volume displacement breast specimens and their relationship to the breast than that for simple breast excisions and mastectomies. Oncoplastic specimens generally have irregular shapes and the skin excision patterns are often more complicated than those encountered in conventional oncologic breast excision specimens. Many oncoplastic specimens have multiple components that are related to one another anatomically (Fig. 3). Therefore, the initial step in evaluating an oncoplastic specimen is to confirm the nomenclature. A large volume displacement oncoplastic breast specimen is defined by the design of the skin excision and the location of the vascular pedicle remaining in the breast, which provides perfusion to the nipple areola complex (Figs. 1 and 2). The type of specimen must be confirmed by inspection and by communication with the surgeon before proceeding with dissection and sectioning. The simplest specimen is termed an "inferior pole excision" from superior pedicle circumvertical skin incision pattern that is associated with a superior pedicle (Fig. 1a). Note that the skin surrounding the nipple areola is removed separately in order to facilitate final closure of the incisions and provide a breast lift. Fig. 2a illustrates the "inverted-T (Wise) pattern" excision with a superomedial pedicle. This example illustrates all of the components within a single specimen. Inferior pedicle excisions may be accomplished with either separate specimens or by a single specimen (Fig. 2b,c). In either case, the pedicle separates the medial and lateral wings and the superior keyhole is connected to the inferior portions of the specimen by medial and/or lateral walls.

Once the identity and nomenclature of the specimen is confirmed, locating the precise tumor site is the next step. A specimen radiograph generally accompanies the primary excision specimen and must be reviewed to be certain that the images are consistent with the specimen (Fig. 4). The localizing guide wire (in this example of a left breast specimen) may still be in the specimen or may have been removed prior to delivery to pathology. In many cases, a hypodermic needle has been placed in the specimen by the radiologist in order to guide the pathologist to the target lesion. If a biopsy site clip is visible in the specimen radiograph, its location will generally correspond to the guide needle placed by the radiologist. Any uncertainty regarding these features within the specimen must be resolved with the surgeon and radiologist before proceeding with specimen dissection. This communication, especially with large volume displacement oncoplastic surgical specimens, cannot be understated as miscommunication with regards to orientation can lead unnecessary completion mastectomy outcomes especially if positive margins are present.

At this point, the specimen margins are inked and the specimen is photographed. Photographic documentation of the specimen is strongly recommended before dissection. This provides a reference for the relationship of the primary 
Fig. 4 Oncoplastic breast specimen a and accompanying specimen radiograph $\mathbf{b}$. This specimen is from a left breast extended superomedial pedicle inverted-T (Wise) skin pattern oncoplastic excision and contains a contiguous superior keyhole, lateral wall, and a small lateral wing. The localization wire and a needle placed by the radiologist are in the superior keyhole and identify the tumor
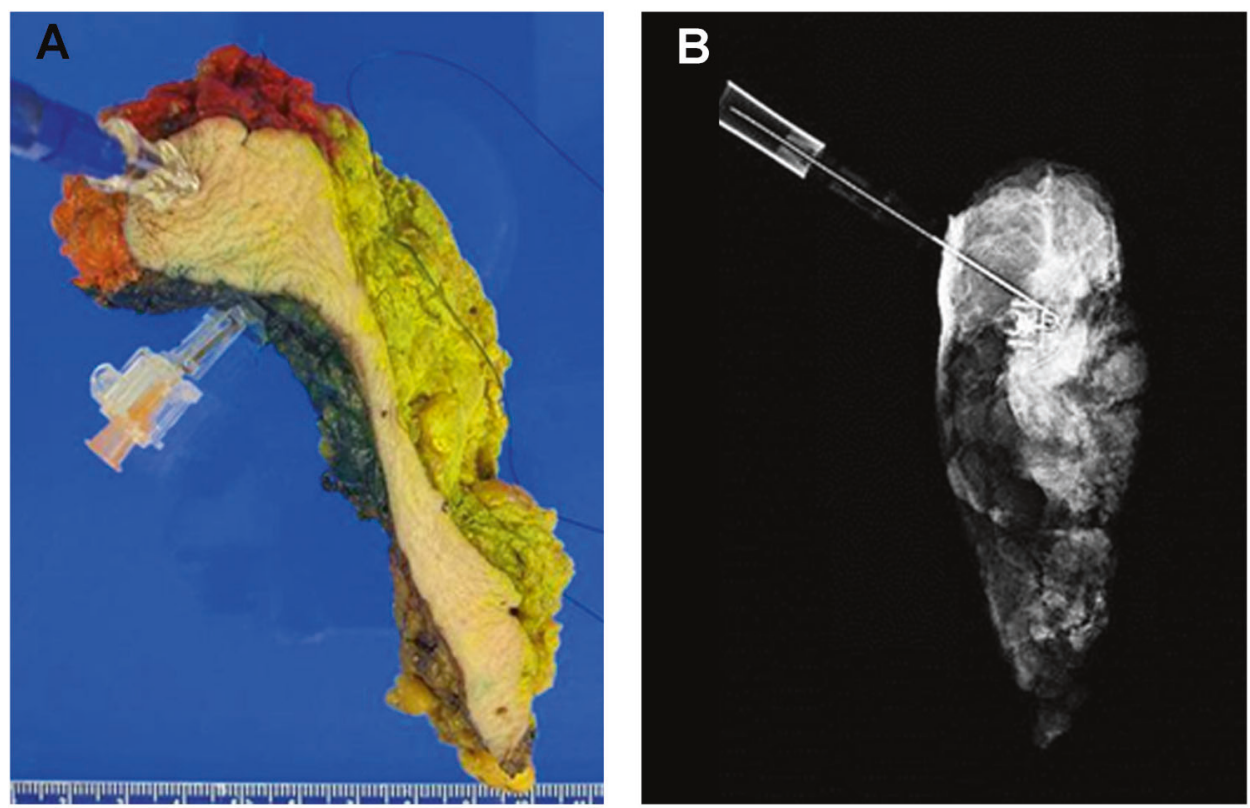

specimen to the in vivo vascular pedicle and to additional specimens generated by the surgical procedure. The photograph also provides a template for a printed or a drawn diagram for documenting the location of histologic sections during microscopic evaluation of the case. A sample diagram for documenting the section location is illustrated in Fig. 5.

\section{Sectioning the specimen}

With the target (tumor) site identified, its location in the specimen should be described precisely. Depending on the specimen pattern, the tumor location may include any of the following: superior keyhole; lateral wing; medial wing; inferior pole; and more rarely the medial or lateral walls. It is essential to identify the margin nearest the tumor with particular attention paid to margins abutting the vascular pedicle remaining in the breast. This determines how the specimen will be sectioned with the focus on preserving the relationship of the tumor to critical margins. Most often, the specimen is sectioned into consecutive slices for visualizing the tumor; however, the direction of the slices may vary in different regions of the specimen. This places emphasis on the need for photographs and section maps to provide critical documentation of section locations.

Taking sections of the tumor with margins must be done with precise description of the location of the sections. In general, most or all of the tumor tissue is submitted for histologic examination and, where possible, it is best to include at least the periphery of the tumor with the nearest margin. It is essential to sample the specimen thoroughly along margins that abut the vascular pedicle. These sections should be clearly defined by their location (e.g., "medial margin of lateral wall" or "inferior margin of superior keyhole", etc.) so that the surgeon can be informed of the precise location in the event of a close or positive margin.

The large mass of many oncoplastic specimens requires that sections be focused on the tumor and nearest margin. Even if the gross tumor is not close to the pedicle, it is recommended that tissue abutting the pedicle is thoroughly sectioned to minimize the chance of missing occult invasive or intraductal carcinoma. Sampling tissue from components of the procedure that do not contain tumor is focused on margins related to tumor-containing components and to the pedicle. Otherwise tissue sampling is similar to that which is done in breast quadrants remote from the tumor in conventional mastectomies. Most oncoplastic procedures are accompanied by a contralateral symmetry mastopexy specimen. The pathologic evaluation for these specimens is performed in the same manner as any contralateral breast specimen in a woman with known breast cancer. The need for follow-up surgical management depends largely on the status of the margins. Future studies evaluating the outcome of large volume oncoplastic breast surgery will be aided by the use of standardized protocols for margin assessment.

\section{Summary}

Our goal in this review was to introduce anatomic terminology known to the breast surgeon; hence spreading and sharing oncoplastic descriptions to the pathology community. With the increasing spread of oncoplastic surgery in 

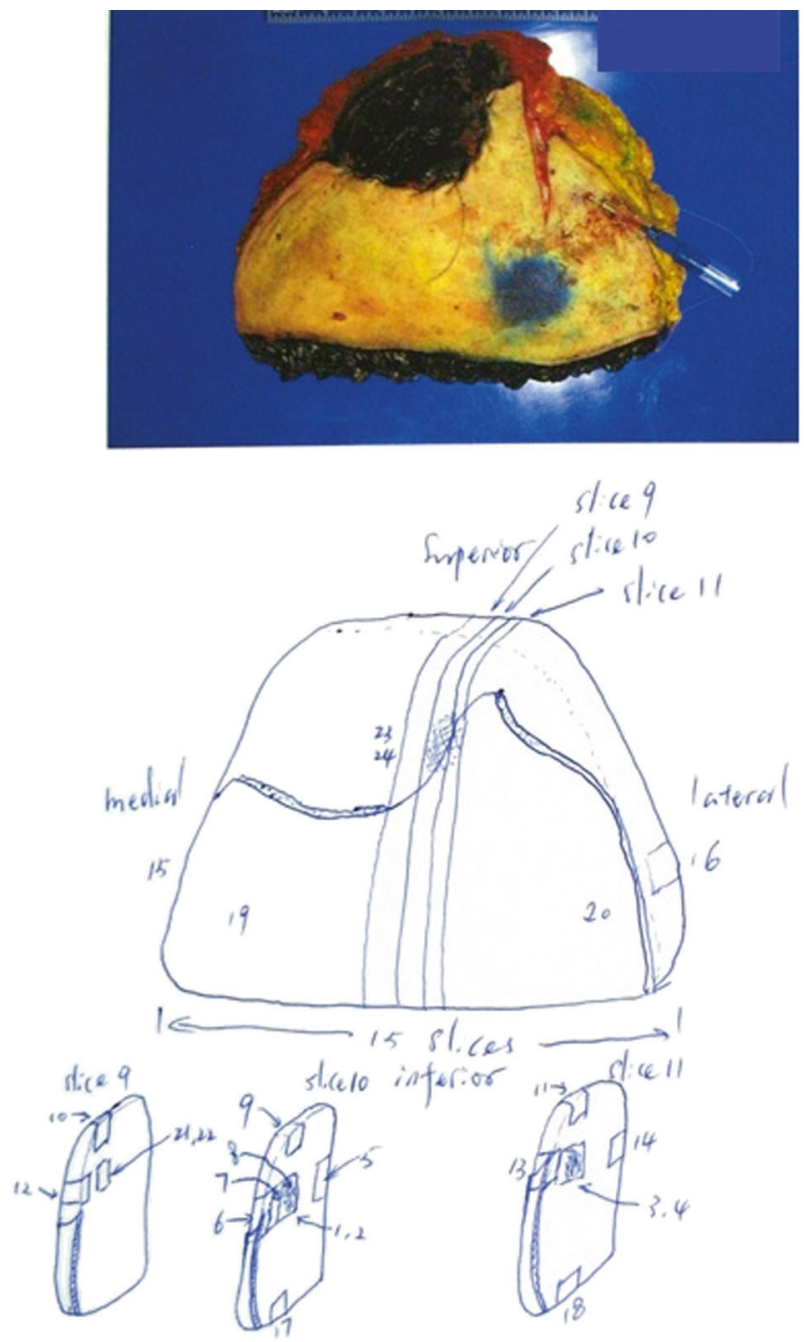

Fig. 5 The specimen is an inferior pole oncoplastic specimen with a localization wire and sentinel lymph node dye injection site. An accompanying hand-drawn diagram is illustrative of how sections taken for microscopic examination may be documented

the United States, it is imperative for pathologists to have a clear understanding how an oncoplastic specimen may present and what a breast oncoplastic surgeon may need to know with regard to positive margins. The procedures presented in this paper highlight and describe one reasonable way to document large volumen breast oncoplastic specimens and call attention to the critical need for communicating promptly with the breast surgeon when any uncertainty arises when assessing an oncoplastic specimen.

The gross examination of breast specimens has been evolving over the past few decades. Accurate gross examination now often relies on the cooperation of pathologists, radiologists, and surgeons [21]. Understanding the correct anatomy and orientation of the breast specimens is very important information to provide the pathologists for identifying and inking the specimen margins. Most breast specimens are oriented by the surgeon. This is usually done by placing two perpendicular sutures on the specimen (e.g., short suture-superior, long suture-lateral). In large volume displacement oncoplastic surgery, accurately locating the margin of each section is essential for patient care especially in the setting of a positive margin where clear understanding regarding location of this positive margin could make the difference between a re-excision partial mastectomy versus a completion mastectomy.

The advantages for the patient provided by large volume reduction oncoplastic surgery must not be compromised by improper evaluation of the surgical pathology specimen. When the final closure of the surgical incisions is accomplished, regions of the breast that were previously distant from one another are now in apposition to one another. It is essential that the tissue margins forming the edges of such closures have been adequately and precisely evaluated in the event a positive margin is found. The amount and location of residual tumor is crucial information for the surgeon in determining the need for further surgical or radiation therapy. Such a decision ultimately rests on the pathologist's determination of the presence of tumor and documentation of tumor location in the histologic sections. Although oncoplastic breast specimens can be more complex than conventional breast excisions, the principles of pathologic examination remain the same. What differs with oncoplastic specimens is that the pathologist must use standard specimen nomenclature and anatomic orientation when identifying and reporting the pathologic findings. We have found that the most important factor for facilitating the pathologic evaluation and processing of oncoplastic breast specimens is unambiguous communication with the surgeon from the beginning so that the type of specimen(s) and the orientation is confirmed. Such communication possibly expands surgical options when the unfortunate circumstances of positive margins occur. Historically, positive margins (no tumor on ink for invasive breast cancer [22]; $<2 \mathrm{~mm}$ for ductal carcinoma in situ [23]) in oncoplastic surgery meant that the patient subsequently underwent a completion mastectomy [15]. With clear understandings in specimen orientation and processing, modern oncoplastic surgical options when met with positive margins may provide an option for additional shaved margin(s) and possibly still allowing the breast cancer patient the breast conservation option assuming the patient has enough breast tissue for additional shaved margins [24, 25]. Clearly, the pathologist's role is central to this process. Our objective is to introduce the standard surgical nomenclature for oncoplastic breast specimens to pathologists, propose guidelines for evaluation of these specimens, and to promote their incorporation into surgical pathology reports (Table 1). Hopefully, our experience with these specimens will provide basic working guidelines for their handling and evaluation and for instructing pathologists in training. 
Table 1 Pathologic examination of breast large volume oncoplastic surgical specimens

1 Confirm specimen nomenclature, orientation, and tumor location by direct communication with the surgeon and review of specimen radiographs.

2 Ink margins and photograph specimen(s).

3 Locate tumor/target lesion and determine optimal sectioning for margin evaluation; pay close attention to margins related to pedicle.

4 Document location of histologic sections on photographic print or drawn section map.

5 Use standardized specimen nomenclature in the surgical pathology report; especially when identifying and reporting margins.

Conflict of interest The authors declare that they have no conflict of interest.

\section{References}

1. Institute NC. SEER Stat Fact Sheets: Female Breast Cancer. http:// seer.cancer.gov/statfacts/html/breast.html. 2016. Accessed: 7/ 272016.

2. Losken A, Ghazi B. An update on oncoplastic surgery. Plast Reconstr Surg. 2012;129:382e-3e.

3. Franceschini G, Martin Sanchez A, Di Leone A, et al. New trends in breast cancer surgery: a therapeutic approach increasingly efficacy and respectful of the patient. G Chir. 2015;36:145-52.

4. De La Cruz L, Blankenship SA, Chatterjee A, et al. Outcomes after oncoplastic breast-conserving surgery in breast cancer patients: a systematic literature reaview. Ann Surg Oncol. 2016;23:3247-58.

5. Clough KB, Kaufman GJ, Nos C, Buccimazza I, Sarfati IM. Improving breast cancer surgery: a classification and quadrant per quadrant atlas for oncoplastic surgery. Ann Surg Oncol. 2010;17:1375-91.

6. Clough KB, Ihrai T, Oden S, et al. Oncoplastic surgery for breast cancer based on tumour location and a quadrant-per-quadrant atlas. Br J Surg. 2012;99:1389-95.

7. Audretsch W. Reconstruction of the partial mastectomy defect: classification and method. In: Spear S (ed). Surgery of the breast: principles and art. Philadelphia, PA, USA: Lippincott Raven; 1998, pp. 155-96.

8. Chatterjee A, Dayicioglu D, Khakpour N, Czerniecki BJ. Oncoplastic surgery: keeping it simple with 5 essential volume displacement techniques for breast conservation in a patient With moderate- to large-sized breasts. Cancer Control. 2017;24:1073274817729043.

9. Losken A, Dugal CS, Styblo TM, Carlson GW. A meta-analysis comparing breast conservation therapy alone to the oncoplastic technique. Ann Plast Surg. 2014;72:145-9.

10. Chatterjee A, Losken A, Graham R, et al. Plastic and reconstructive surgery. PPRS PSRC PodiumProofs, Global Open. 2016;4:35-107.

11. Piper ML, Esserman LJ, Sbitany H, Peled AW. Outcomes following oncoplastic reduction mammoplasty: a systematic review. Ann Plast Surg. 2016;76:S222-6.
12. Carter SA, Lyons GR, Kuerer HM, et al. Operative and oncologic outcomes in 9861 patients with operable breast cancer: singleinstitution analysis of breast conservation with oncoplastic reconstruction. Ann Surg Oncol. 2016;23:3190-8.

13. Yang JD, Lee JW, Cho YK, et al. Surgical techniques for personalized oncoplastic surgery in breast cancer patients with smallto moderate-sized breasts (part 1): volume displacement. J Breast Cancer. 2012;15:1-6.

14. Anderson BO, Masetti R, Silverstein MJ. Oncoplastic approaches to partial mastectomy: an overview of volume-displacement techniques. Lancet Oncol. 2005;6:145-57.

15. Losken A, Hart AM, Chatterjee A. Updated evidence on the oncoplastic approach to breast conservation therapy. Plast Reconstr Surg. 2017;140:14S-22S.

16. Chatterjee A. Long term effects of modern breast cancer surgery. Gland surgery 2017:17648. https://doi.org/10.21037/gs.2017.10.09.

17. Spear SL. Surgery of the Breast - Principles and Art. 3rd edn. 2011.

18. van Deventer PV, Graewe FR. The blood supply of the breast revisited. Plast Reconstr Surg. 2016;137:1388-97.

19. Hansen JE. Avoiding the unfavorable outcome with wise pattern breast reduction. Clin Plast Surg. 2016;43:349-58.

20. Barnea Y, Inbal A, Barsuk D, et al. Oncoplastic reduction using the vertical scar superior-medial pedicle pattern technique for immediate partial breast reconstruction. Can J Surg. 2014;57: E134-40.

21. Huo L. A practical approach to grossing breast specimens. Ann Diagn Pathol. 2011;15:291-301.

22. Moran MS, Schnitt SJ, Giuliano AE, et al. Society of surgical oncology-american society for radiation oncology consensus guideline on margins for breast-conserving surgery with wholebreast irradiation in stages $\mathrm{i}$ and ii invasive breast cancer. Int $\mathrm{J}$ Radiat Oncol Biol Phys. 2014;88:553-64.

23. Morrow M, Van Zee KJ, Solin LJ, et al. Society of surgical oncology-american society for radiation oncology-american society of clinical oncology consensus guideline on margins for breast-conserving surgery with whole-breast irradiation in ductal carcinoma in situ. J Clin Oncol. 2016;34:4040-6.

24. Crown A, Wechter DG, Grumley JW. Oncoplastic breastconserving surgery reduces mastectomy and postoperative reexcision rates. Ann Surg Oncol. 2015;22:3363-8.

25. Clough KB, Gouveia PF, Benyahi D, et al. Positive margins after oncoplastic surgery for breast cancer. Ann Surg Oncol. 2015;22:4247-53. 